Bolm. Zool., Univ. S. Paulo

9: $231-257,1985$

\title{
SOBRE RHINODRILUS E UROBENUS (OLIGOCHAETA, GLOSSOSCOLECIDAE)
}

\section{GILBERTO RIGHI}

Departamento de Zoologia, Instituto de Biociências, Universidade de Säo Paulo. (recebido em 29.V.1985)

RESUMO - Os gêneros Rhinodrilus e Urobenus foram separados e suas diagnoses reformuladas. As espécies de Rhinodrilus e de Urobenus foram listadas com seus sinônimos e ocorrências e apresentadas chaves para sua identificação. Quatro espé cies novas foram reconhecidas: $R$. corderoi, $R$. jucundus, $R$. marcusae e $U$. petrerei. $U$. buritis e $U$. brevis foram eleva das ao nivel de espécie. Novas ocorrências e variaçōes foram registradas para $R$. motucu e $R$. xeabaibus.

ABSTRACT - The genera Rhinodrilus and Urobenus were set apart and their diagnoses reformulated. The check-list of Rhinodrilus and Urobenus species with synonyms, occurrences and keys for identification were presented. Four new species were recognized: $R$. corderoi, $R$. jucundus, $R$. marcusae and $U$. petrerei. $U$ buritis and $U$. brevis were raised to species level. New occurrences and variations were indicated for $R$. motucu and $R$. xeabaibus.

O gênero Rhinodrilus (= Rhinodrilus + Urobenus), como è conhecido até agora, contem 42 espécies e subespécies que habitam a América do Sul entre os paralelos de $11^{\circ} \mathrm{N}$ e $300 \mathrm{~S}$, a leste dos Andes. Duas espécies sao conhecidas dos Andes, $R$. sibateensis e $R$. parvus, em regióes drenadas pelas bacias do Rio Orinoco a primeira e do Rio Paraguai a segunda. Neste gênero estão incluidas desde espécies minúsculas como $R$. cor deroi, com $35 \mathrm{~mm}$ de comprimento, até a gigantesca $R$. fafner, com $2.100 \mathrm{~mm}$. Suas espécies exploram grande diversidade de häbitats, havendo desde anfíbias, $R$. duseni, até adaptadas às regiōes secas do cerrado, $R$. alatus. 0 gểnero notabiliza - se também pela complexidade anatómica com suas implicações filogenéticas. Os septos anteriores de algumas espécies são muito delgados, frágeis e por vezes irreconheciveis, em ou tras são muito espessos, musculosos e frequentemente com a origem dorsal deslocada para trás. As glāndulas calcíferas apresentam diferentes tipos de arquitetura, bem como os ne frídios. Notável é a complexidade do sistema circulatório e a variabilidade do aparelho reprodutor. A ültima revisão do gênero è de Michaelsen (1918) que incluiu 8 espécies com se- 
gurança e 2 com dúvidas. Posteriormente foram descritas várias espécies e è de se esperar que muitas outras sejam reco nhecidas de regiōes pouco ou nada estudadas, como o BrasiI central e norte, Guianas, Venezuela, parte oriental dos An des na Colômbia, Peru, Bolívia e o Paraguai. 0 interesse eco -fisiológico e anátomo-filogenético do gênero, o grande núme ro de espécies, o fato da ültima revisão ter sido feita a mais de 60 anos e a necessidade de classificar o material co ligido, tornou necessário este primeiro estudo crítico. Estū dos detalhados da arquitetura das glândulas calcíferas, dos nefrídios e da topografia vascular deveräo conduzir a novos agrupamentos.

Aos colegas e amigos, Drs. Jorge Jim, Miguel Petrere, Edvaldo Rosa dos Santos, Edilson Gouvêa e Mário Grempel, agradeço pela coleta e doação da maioria dos animais aqui tra tados. Os trabalhos foram feitos por meio de dissecçōes $\bar{e}$ cortes histológicos, $10 \mathrm{\mu m}$, corados pelo método tríplice de Mallory (Pantin, 1964). O material está depositado no Departamento de Zoologia da Universidade de São Paulo.

\section{Rhinodrilus Perrier, 1872}

Rhinodrilus Perrier, 1872:65.

Rhinodrilus part.; Benham, 1890:253; Beddard, 1895:636; Michaelsen, 1900b:430; 1918:165; Stephenson, 1930:894; Cordero, 1945:8; Righi, 1971a:10; Jamieson, 1971:738.

Rhinodrilus (Rhinodrilus) part.; Cognetti, 1906:174.

Geogenia part.; Vaillant, 1889:188.

Tykonus part.; Beddard, 1895:650.

Anteus part.; Beddard, 1895:651; Rosa, 1896:90.

\section{Diagnose}

Cerdas dispostas em 4 pares de séries longitudinais re gulares, raramente una ou mais séries irregulares na região posterior. Traves pubertais intraclitelares, raramente fal tam. Moela em VI. Três pares de glândulas calcíferas em VIIIX, todas de estrutura semelhante, tubular-dicotômica (paniculada) ou tubular composta. Vaso subneural presente. Holândrico, em geral cleistorquídico. Vesículas seminais curtas, na maioria limitadas ao próprio segmento. Poros masculinos intraclitelares, 1 par, raramente 2 pares. Metagínico. Esper matecas presentes, intracelómicas, raramente intraparietais.

Tipo do gênero

$$
\text { Rhinodrilus paradoxus Perrier, 1872: }
$$

\section{Consideraçōes}

O gênero Rhinodrilus ficou restrito às espécies em que os 3 pares de glândulas calcíferas apresentam o mesmo tipo de organização, tubular-dicotômico (Rispenschlauchtasche de Michaelsen, 1918) ou tubular-composto (Kompositenschlauch tasche). A distinção entre os 2 tipos estruturais não è precisa, havendo formas intermediárias como $R$. marcusae. Segun- 
do Michaelsen (1926) as glândulas de $R$. garbei são do tipo alveolar (Wabentaschen), que è uma variedade de tubular-com posta com cavidade central longa. No geral as glândulas cal= cíferas de Rhinodrilus têm uma capa muscular que pode ser ora mais ora menos espessa, possibilitando sua maior ou me nor contração. Assim, numa glândula de estrutura tubular-com posta ou alveolar com cavidade central longa e estreita, túbulos calcíferos inclinados e parede contraída, não è fácil a distinção entre a cavidade central e os túbulos calcífe ros, especialmente em animais não bem fixados para estudos histologicos. Frequentemente o tipo tubular-dicotômico encon tra-se em glầndulas calcíferas piriformes e de origem dorsal no esôfago e o tipo tubular-composto em glândulas reniformes e de origem lateral.

Lista das espécies com sinonímia e ocorrências.

1. Rhinodrilus curtus Stephenson, 1931 .

Rhinodrilus curtus Stephenson, 1931:81, figs. 26-27

Ocorrência - Trinidad: Mt.Tucouché (Stephenson, 1931).

2. Rhinodrilus paradoxus Perrier, 1872.

Rhinodrilus paradoxus Perrier, 1872:66, est. 1, figs. 9-12 ;

(part.: subsp. typicus + subsp. appuni part.: sin.var. maus8i) Michaelsen, 1900b:431, 432; (part.) Cognetti, 1906:175; Michaelsen, 1918:177, est. 2, figs. 19-20; Omodeo, 1955:202.

Rhinodrilus paradoxus + R. callichaetus; Beddard, 1895:639, 642 .

Anteus cailichaetus Michaelsen, 1892:220, est. 8, figs. 7-8. Anteus calliohaetus var. maussi (part.) Michaelsen, 1895:24. Anteus paradoxus + A. callichaetus; Rosa, 1896:107, 113.

Ocorrência - Venezuela, Distrito Federal: Caracas (Per rier, 1872; Michaelsen, 1892), El Junquito (Omodeo, 1955); Es tado Carabobo: Puerto Cabello (Michaelsen, 1895)

3. Rhinodrilus romani Michaelsen, 1928 .

Rhinodrilus romani Michaelsen, 1928:1.

Ocorrência - Brasil, Estado Amazonas: entre S. Felipe e S. Gabriel (curso superior do Rio Negro) (Michaelsen, 1928)

4. Rhinodrizus senex Righi, 1984.

Rhinodrilus senex Righi, 1984b:244, figs. 1-2.

Ocorréncia - Venezuela, Estado Aragua: Parque Nacional Hen ri Pittier: Rancho Grande (Righi, 1984b). 
5. Rhinodrilus elisianae Righi et all. 1976. Rhinodrizus elisianae Righi et all. 1976:358, figs. 43-45. Ocorrência - Brasil, Estado Amazonas: Sucunduri (Righi et all. 1976)

6. Rhinodrilus curiosus Righi et all. 1976. Rhinodrilus curiosus Righi et all. 1976, figs. 38-42.

Ocorréncia - Brasil, Estado Amazonas: Estrada Manaus-Itacoatiara, Km 26 (Reserva Ducke) (Righi et all. 1976)

7 Rhinodrilus contortus Cernosvitov, 1938.

Rhinodrilus contortus Cernosvitov, 1938:401, figs. 1-11.

Ocorrência - Brasil (Cernosvitov, 1938).

8. Rhinodrilus appuni(Michaelsen, 1892).

Anteus appuni Michaelsen, 1892:218; Rosa, 1896:113.

Anteus appuni + A. callichaetus var. maussi (part.) Michaelsen, $1895: 19,20$, figs. 1-3.

Tykonus appuni; Beddard, 1895:651.

Rhinodrilus paradoxus appuni (part.: excl. sin. Anteus cal lichaetus var. sieversi e var. maussi part.); Michaelsen, $1900 \mathrm{~b}: 432$.

Rhinodrilus (R.) paradoxus (part.); Cognetti, 1906:175. Rhinodrilus appuni; Michaelsen, 1918:173.

Ocorrência - Venezuela, Estado Carabobo: Puerto Cabello (Michaelsen, 1892; 1895)

9. Rhinodrizus sieversi (Michaelsen, 1895)

Anteus callichaetus var. sieversi Michaelsen, 1895:23. Rhinodrilus paradoxus appuni (part.: sin. Anteus callichae tus var. sieversi); Michaelsen, 1900b:432.

Rhinodrilus (R.) paradoxus (part.); Cognetti, 1906:175. Rhinodrilus sieversi; Michaelsen, 1918:170; 1935:52.

Ocorrência - Venezuela, Estado Carabobo: Puerto Cabello (Michaelsen, 1895) Trinidad: Mt. Tucucho (provavelmente Mt. Tucouchê) (Michaelsen, 1935)

10. Rhinodrilus torquemadai Righi, 1984. Rhinodrilus torquemadai Righi, 1984c:199, figs. 10-14.

Ocorrências - Brasil, Estado Mato Grosso: Cáceres, Pontes e Lacerda, Vila Bela da Santíssima Trindade (Righi, 1984c). 
11. Rhinodrilus mamita Cordero, 1943.

Rhinodrilus mamita Cordero, 1943:3. 1943)

Ocorrência - Brasil, Estado Ceará: Maranguape (Cordero,

12. Rhinodrilus francisci Cordero, 1944.

Rhinodrilus francisci Cordero, $1944 \mathrm{a}: 1$, fig. 1.

Ocorrência - Brasil, Estado Pernanbuco: Município de Floresta: Sabiucá (Cordero, 1944a).

\section{Rhinodrilus senckenbergi Michaelsen, 1931.}

Rhinodrilus senckenbergi Michaelsen, 1931:83, figs. 3-4.

Ocorrência - Brasil, Estado Espírito Santo: região do Rio Doce (Michaelsen, 1931)

14. Rhinodrilus Zongus Cernosvitov, 1934.

Rhinodrilus longus Cernosvitov, 1934:54, figs. 16-18; 1935 :

21, figs. 44-49.

Ocorrências - Guiana Francesa: morros na região do curso inferior do Rio Mahury. Brasil, Território do Amapá: curso inferior do Rio Calçoene (Cernosvitov, 1934;1935).

15. Rhinodrilus fuenzalidae Cordero, 1944.

Rhinodrilus fuenaalidae Cordero, 1944b: 1.

Ocorrência - Venezuela, Estado Miranda: El Guayabo (Corde ro, $1944 \mathrm{~b})$.

16. Rhinodrilus corderoi, sp. n.

Rhinodrilus fuenzalidae; Righi, 1984b:246, figs. 3-6.

Ocorrência - Venezuela, Estado Carabobo: San Esteban (Righi, 1984b)

\section{Rhinodrilus sibateensis (Michaelsen, 1900)}

Anteus sibateensis Michaelsen, 1900a:247. Rhinodrilus sibateensis; Michaelsen, 1900b:433; 1918:193.

Ocorrência - Colômbia, Departamento Cundinamarca: Sibaté (próximo de Fusagasugá) (Michaelsen, 1900a). 
18. Rhinodrizus Zakei Michaelsen, 1934.

Rhinodrilus lakei Michaelsen, 1934:9, figs. 4-8.

Ocorrências - Brasil, Estado Roraima: curso superior do Rio Catrimani (afluente do Rio Negro, ca. $2^{\circ} \mathrm{N}, 63-62{ }^{\circ} \mathrm{W}$ ); Estado Amazonas: arredores de Manaus (Michaelsen, 1934).

19. Rhinodrizus jucundus, sp. n.

Ocorrência - Brasil, Estado Goiás: Paraíso do Norte.

20. Rhinodrilus priollii Righi, 1967

Rhinodrilus priollii Righi, 1967:475, figs. 1-6.

Ocorrência - Brasil, Estado Amazonas: Manaus (Righi,1967)

21. Rhinodrilus annulatus Cernosvitov, 1934.

Rhinodrilus annulatus Cernosvitov, 1934:54, figs. 19-20;

1935:23, figs. 50-53.

Ocorrência - Venezuela: Haute Carsevenne (Cernosvitov, 1934; 1935). Provavelmente Brasil, Território do Amapá: curso superior do Rio Calçoene.

22. Rhinodrilus mortis Righi, 1972 .

Rhinodrilus mortis Righi, 1972b:157, figs. 7-12.

Ocorrência - Brasil, Estado Mato Grosso: São José da Serra (Righi, 1972b).

23. Rhinodrilus lucilleae Righi et all. 1976.

Rhinodrilus lucilleae Righi et all. 1976:354, figs. 33-37 1976)

Ocorrēncia - Estado Amazonas: Sucunduri (Righi et all.

24. Rhinodrilus duseni Michaelsen, 1918.

Rhinodrilus duseni Michaelsen, 1918:190; Righi, 1971a:16,

figs. 17-22; 1974:555; 1984a:101; Schroeder-Araujo,

1978:163, figs. 1-9.

Ocorrências - Brasil, Estado Paraná: Curitiba (Michael sen, 1918); Estado São Paulo: Pilar do Sul (Righi, 197la), I tapeva (Righi, 1974) Estado Mato Grosso: Fátima do Sul (Ri= ghi, $1984 \mathrm{a}$ ) 
25. Rhinodrilus adelae Cordero, 1943.

Rhinodrilus adelae Cordero, 1943:1.

Ocorrência - Brasil, Estado Ceará: Açude Acarapé do Meio (Cordero, 1943).

26. Rhinodrilus bursiferus Righi, 1971.

Rhinodrilus bursiferus Righi, I97la:13, figs. 13-16.

Ocorrência - Brasil, Território do Amapá: Serra do Navio, margens do curso médio do Rio Amapari (Righi, 1971a:13).

27 Rhinodrilus evandroi Righi, 1971.

Rhinodrizus evandroi Righi, 197la:17, figs. 23-25.

Ocorrência - Brasil, Distrito Féderal: Brasilia (Righi, 1971a).

28. Rhinodrilus garbei Michaelsen, 1926 .

Rhinodrilus garbei Michaelsen, 1926:274.

Ocorrência - Brasil, Estado Minas Gerais: Pirapora; Estado São Paulo: Boturata (provavelmente Botucatu) (Michaelsen, 1926 ).

29. Rhinodrilus motucu Righi, 1971.

Rhinodrilus motucu Righi, 197la:21, figs. 29-34; 1972:257

Rhinodrilus motucu unais Righi, 1971b:7, figs. 5-8.

Rhinodrilus motucu cuiabanus Righi, 1984a:l01, figs. 35-40.

Ocorrência - Brasil, Estado Mato Grosso: Cuiabá, Poconé (Righi, 1984a); Estado Goiās: Porangatu (Righi, 197ía), NE de Uruaçu na margem do Rio Tocantins; Estado Bahia: Una, Ita gibá (não Itajuba) (Righi, 1971b), Jequié. Estado Sergipe : Umbauba.

30. Rhinodrilus marcusae, sp. n.

Ocorrência - Brasil, Estado Bahia: Porto Mandioca no Rio Paraguaçu.

31. Rhinodrilus hoefiingae Righi, 1980.

Rhinodrilus hoeflingae Righi, 1980b:7, figs. 8-14

Ocorrências - Brasil, Estado Minas Gerais: Caetanópolis, Cachoeira dos Macacos (Righi, 1980b) 
32 Rhinodrilus xeabaibus Righi, 1969.

Rhinodrilus xeabaibus Righi, 1969:155, fig. 4.

Ocorrência - Brasil, Estado Rio de Janeiro: Parque Nacional do Itatiaia: Maromba (Righi, 1969), Mauã.

33. Rhinodrilus alatus Righi, 1971.

Rhinodrizus alatus Righi, 1971a:10, figs. 10-12; 1972a:257;

H8fling, 1980:22, figs. 1-24; 1982:297, figs. 1-11.

Ocorrências - Brasil, Estado Minas Gerais: Sete Lagoas, Paraopeba (Righi, 197la)

34. Rhinodrilus (?) proboscideus G. Schneider, 1892.

Rhinodrilus proboscideus G. Schneider, 1892:42; Beddard,

$1895: 642$; (sp. dubiae) Michaelsen, 1900b:469

Anteus proboscideus; Rosa, 1895:114.

Ocorrência - Trinidad (G. Schneider, 1892)

35. Rhinodrilus (?) horsti (Beddard, 1892).

Anteus gigas; Horst, 1891:77, est. 6. figs. 1-4.

Anteus horsti Beddard, 1892:117; 1895:652; Rosa, 1896:111.

Rhinodrilus horsti; Michaelsen, 1900b:430; 1918:194.

Rhinodrilus (R.) horsti; Cognetti, 1906:174.

Ocorrência - Brasil (Horst, 1891)

36. Rhinodrilus (?) parvus (Rosa, 1895).

Anteus parvus Rosa, 1895:2; $1896: 104$.

Rhinodrilus parvus; Michaelsen, 1900b:431; Cognetti, $1902: 4$. Rhinodrilus (R.) parvus; Cognetti, 1906:175.

Rhinodrilus (?) parvus; Michaelsen, 1918:170.

Ocorrências - Argentina, Província Chaco: Resistência (Ro sa, 1895). Bolivia, Departamento Potosí: Caiza (Cognetti 1902)

37. Rhinodrilus (?) fafner Michaelsen, 1918.

Rhinodrilus (?) fafner Michaelsen, 1918:195.

Ocorrência - Brasil, Estado Minas Gerais (Michaelsen 1918). 
Chave para as espécies de Rhinodrilus.

1 - Dois pares de espermatecas.

-- Três ou mais pares de espermatecas, por vezes intraparietais.

2 - Poros das espermatecas em $6 / 7$ e $7 / 8$. .R. curtus

-- Poros das espermatecas em $7 / 8$ e $8 / 9$.

3 - Todos os septos frágeis que os seguintes. $\dot{ }$ iो em $\ddot{X} \dot{X}-\ddot{X} \dot{X} \dot{I}, a b$.

4 - Um par de traves pubertais em XX-XXII, $a b$. paradoxus

--- Traves pubertais ausentes...

5 - Poros das espermatecas na linha $c . \quad . R$. romani

-.- Poros das espermatecas na linha $a b$. ... . senex

6 - Espermatecas com as cavidades da ampola e do duto subdivididas em câmaras intercomunicantes

-- Espermatecas com $\ddot{\text { as cavidades da ampola e do }{ }^{R} \text { torquemada } i}$ simples, pode haver câmaras seminais na parede do du to.

7 - Dutos das espermatecas sem cầmaras seminais....

. ..R. elisianae

--- Dutos das espermatecas com câmaras seminais.

8 - Poros das espermatecas próximos dá linha MV - . . . . . R. curiosus

--- Poros das espermatecas na i inha cd..

9 - Clitelo em XVII-XXIV Esôfago anterior enrolado Glāndulas calciferas com apendice ental.

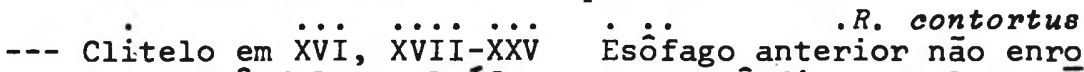
lado. Glândulas calcíferas sem apêndice ental.

10 - Na região posterior $a a=12 a b$.

. $R$. appuni

-.. Na região posterior $a a=2 a b$.

R. sieversi

11 - Três pares de espermatecas, tódas ou só os 2 últimos

pares salientes na cavidade do corpo. rietais.

--- Quatro ou mais pares de espermatecas, todas intrapa-

12 - Poros das espermatecas em $5 / 6-7 / 8$.

-.-- Poros das espermatecas em 6/7-8/9.

13 - Nefridióporos em $a b$.

-.-- Nefridióporos em cd.

14 - Espermatecas sem câmaras seminais.

-.-- Espermatecas com câmaras seminais...

... mamita
. R. francisci

13

16

15 - Comprimențo dos animais menor que $10 \mathrm{~cm}$. - : : : $\cdot R$. senckenbergi

--- Comprimento dos animais maior que $30^{\circ} \mathrm{cm}$. . $R$. Zongus

16 - Cerdas dispostas em séries longitudinais regulares ao longo de todo o corpo. . . . .

--- Na região posterior uma ou mais séries de cerdas dis postas irregularmente. … : : . . ...

17 - Espermatecas com câmaras seminais. : $\cdot$. hoefïingae

--- Espermatecas sem câmaras seminais. ...

18 - Comprimento dos animais $128-150 \mathrm{~mm}$. Na regiäo posterior cerdas $b$ com disposiçāo irregular, as demais re 
gulares.

.R. fuenzalidae

Comprimento dos animais $35-44 \mathrm{~mm}$. Na região posteri or só as cerdas a têm disposição regular, as de mais irregulares. : $\quad . R$. corderoi

19 - Espermatecas sem câmaras seminais.

..- Espermatecas com câmaras seminais.

20 - Traves pubertais en $1 / 2$ XXIII - $1 / 2$ XXVIII.

...R. annuiatus

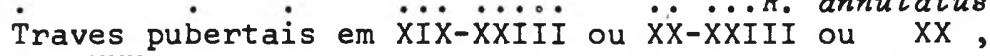
XXI-XXV

21 - $\begin{aligned} & \text { Comprimento dos animais menor que } \dot{15} \mathrm{~cm} \text {. Na regiäo } \\ & \text { posterior } a a=a b . .\end{aligned}$ posterior $a a=a b . \dot{ }$.
Comprimento dos animais maior que $3 \dot{0} \mathrm{~cm}$. sibateensis $\mathrm{Na}$ região posterior $a a>a b$. ....... . ....

22 - Traves pubertais em XIX-XXIII. ....... . prioli $i_{i}$

--- Traves pubertais em XXI, XXI - i/n XXV .

23 - Na regiao posterior $a a=2 a b$. Cerdas genitais onna mentadas com 14 marcas por série. R. lake $\vec{i}$

-.- Na regiāo posterior $a a=6 a b$. Cerdas genitais onna mentadas com 6-7 marcas por série. $R$. jucundus

24 - Campo genital masculino com intersegmentos 20/21, $21 / 22$ e ou não $22 / 23$ muito deprimidos na linha $b$ ou $a b$.

--- Campo genitai mascuilino com intersegmentos simples, sem depressões específicas. 25 - Poros masculinos, 1 par em 20/21. .. . dusen $i$

--- Poros masculinos, 2 pares em XX ou XXI.

26 - Poros masculinos em XXI. Espermatecas com ampola.. .. . . . R. mortis

---- Poros masculinos em $\dot{x} \dot{X}$. Éspermatecas sem ampola.

- $\quad \cdots \quad \ldots R$. Iucilieae 27 - Campo genital masculino sem traves pubertais.

-.- Campo genitai masculino com traves pubertais. adelae

28 - Esôfago com câmaras dorsais em VII-IX. Espermatecas com ampola diminuta, no máximo $1 / 5$ do comprimento do duto. $\quad . \quad$. bursiferus Esôfago sem cầmaras dorsais. Espermatecas com ampola de comprimento igual ou maior do que o duto.

Traves pubertais em $1 / 2$ XX - $1 / 2$ XXIII ( $=3$ segmentos). . $\quad \cdots \quad \ldots . . . . . . . R$ evandroi

--- Traves pubertais em $\dot{i} / \mathrm{n}$ XXI, XXI $\dot{X} \dot{X} \ddot{X X V} \dot{I}, I / n$ XXVII

30 - Campo genital masculino com faixa glandular elevada, com forma de $I$, mediana ventral em XXI-XXVII.

--- Campo genital masculino sem faixa glandular como a-

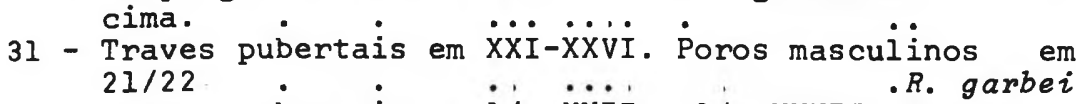
Traves pubertais em $i / n$ XXII - $1 / n$ XXVII. Poros mas culinos em $23 / 24 \ldots$. . . . R. motucu

32 - Espermatecas em 6/7-14/15. Traves pubertais temi nam em XXVII. $\quad R$. (?) fafner 
--- Espermatecas em 6/7-9/10, 10/11. Traves pubertais terminam em XXVIII, XXX. . . .

33 - Espermatecas em 6/7-9/10. Traves pubertais em $2 / 3$ XX $-2 / 3$ XXVIII.... .... ... . R. xeabaidus

-.- Espermatecas em 6/7-10/11. Traves pubertais em $1 / \mathrm{n}$ XXIII $-1 / \mathrm{n} X X X$.

Rhinodrilus (?) proboscideus, R. (?) horsti e R. (?) parvus não constam da chave por serem insuficientemente co nhecidas.

Rhinodrilus corderoi,sp. n.

Rhinodrilus fuenzalidae; Righi, 1984b:246, figs. 3-6.

Considerações

Confrontando as descriçōes de $R$. fuenzalidae Cordero (1944b) e Righi (1984b), concluo tratarem-se de 2 espécies diferentes como demonstro abaixo. Para a espécie de Righi eu dou o nome $R$. corderoi em memória do Prof. Dr. Ergasto H. Cordero.

$R$. corderoi distingue-se de $R$. fuenzalidae pelos se guintes caracteres: $R$. corderoi - comprimento $35-44 \mathrm{~mm}$ ( $R$. fuenzalidae - comprimento $128-150 \mathrm{~mm}$ ); na região posterior do corpo as cerdas a têm disposição regular, as demais ir regulares (na regiāo posterior do corpo as cerdas $b$ têm dis posição irregular, as demais regulares); campo genital masculino com um par de traves pubertais largas em $1 / 2 \mathrm{XX}-1 / 2$ XXIII (campo genital masculino com um par de sulcos longitudinais em $1 / 2 \mathrm{XX}-1 / 2 \mathrm{XXIII)}$

Rhinodrilus jucundus, sp. n. (Figs. 1-2)

Material

Brasil, Estado Goiās: Estrada Brasília-Belēm, $30 \mathrm{Km} \mathrm{N}$ de Paraíso do Norte (ca. $10^{\circ} 10^{\prime} \mathrm{S}-488^{\circ} 3^{\prime} \mathrm{W}$ ), 1 clitelado (ZU819), G.Righi col. 8.I.1967

\section{Descriçāo}

0 animal está fragmentado em 4 partes. 0 fragmento anterior mede $103 \mathrm{~mm}$ de comprimento e tem 74 segmentos, os demais fragmentos medem 70,12 e $30 \mathrm{~mm}$ e o número de segmentos é 115,26 e 49 respectivamente. Assim o comprimento total é de $215 \mathrm{~mm}$ e $\circ$ número de segmentos 264. 0 diámetro no clitelo é $8,4 \mathrm{~mm}$, na região média $6,1 \mathrm{~mm} e$ na regiāo posterior 9,0 $\mathrm{mm}$. A cor geral do corpo na região posclitelar é amarela azu lada, como o n! 233 de Séguy (1936), na regiāo preclitelar é marrom clara como o no 694 . Na região anterior a segmentaçāo externa nāo corresponde à interna, de modo que a segmen- 
taçāo referida a seguir foi estabelecida admitindo-se a posi ção da moela no segmento VI. No interior da cavidade bucal, aberta por un corte sagital, o prostómio aparece como um dedo curto e largo, com faixas longitudinais partindo da altura de III externo, que, internamente, na cavidade bucal, deve corresponder a I invaginado e irreconhecível. 0 II, invaginado na maior parte, aparece na superfície com $1 / 3$ do comprimento de III. Os segmentos II - I/2 IV são percorridos por numerosos sulcos'longitudinais, os demais segmentos têm a superfície lisa. As cerdas dispöem-se em 8 séries longitudinais regulares, iniciando-se as ventrais em VII e as laterais em VIII. As cerdas normais são sigmóides, alongadas, com nódulo bem diferenciado e ligeiramente distal. Seu $1 / 4$ api cal é ornamentado por marcas semilunares largas e dispostas em 4 séries longitudinais alternas; em cada série há 5-8 mar cas, em geral 6-7. Na regiäo média do corpo o comprimentō das cerdas ventrais varia de 768-867 $\mu \mathrm{m}$, média $818 \mu \mathrm{m}$, e das laterais 596-695 $\mu \mathrm{m}$, média $650 \mu \mathrm{m}$. Na regiäo posterior o com primento das cerdas ventrais varia de $728-854 \mu \mathrm{m}$, média $80 \overline{9}$ $\mu \mathrm{m}$, e das laterais 675-76I $\mu \mathrm{m}$, média $715 \mu \mathrm{m}$. As cerdas ventrais de XIII, XVI e XVIII-XXV säo transformadas em cerdas genitais. As de XIII e XVI estão quebradas, as demais são re tas, com pequena curvatura na extremidade proximal. Seus $3 / \overline{5}$ apicais sao ornamentados por 4 séries alternas de marcas semilunares largas, contando-se 6-7 marcas por série. 0 compri mento destas cerdas varia de $1,32-1,53 \mathrm{~mm}$, média $1,40 \mathrm{~mm}$, o diâmetro proximal varia de 52-68 $\mathrm{\mu m}$, média $57 \mathrm{\mu m}$. As relações entre as cerdas são na região média do corpo (segmentos L-LX) $a a: a b: b c: c d: d d=7,85: 1,00: 12,55: 0,71: 30,28$ e na região posterior (segmentos CCXXX-CCXL) $a a: a b: b c: c d: d d=6,08: 1,00$ : $6,41: 0,60: 16,00$.

0 clitelo ocupa os segmentos $1 / 2$ XIV - $1 / 2$ XXVI (=12), é anelar e bastante túrgido em XV-XXV, com sulcos interseg mentares nitidos, e pouco espesso e aberto ventralmente em $1 / 2$ XIV e 1/2 XXVI. Duas espessas almofadas glandulares ovói des, com ligeira incisäo mediana anterior (Fig. 1) situam-sē ventralmente em XIII e XVI, onde estendem-se por todo o com primento desses segmentos e lateralmente atingem $1 / 3 \mathrm{bc}$. 0 clitelo e as almofadas glandulares são de cor violeta escura como o no 86 de Séguy (1936) Área diferenciada, circular e plana envolve cada cerda genital. As de XIII e XVI estão fundidas e as de XIX circundadas por uma área despigmentada. Um par de traves pubertais pouco elevadas situa-se em XX $1 / 2$ XXV; seus limites são bem demarcados, o lateral é reto e o medial, imediatamente ao lado da linha $b$, é convexo no equador de cada segmento. 0 1/2-2/3 laterais das traves tem cor branca cremosa e o restante medial tem cor semelhante à do clitelo.

Os septos anteriores têm a forma de longos cones inter penetrados, que diminuem sucessivamente até $14 / 15$, os demais são planos. O primeiro septo é $05 / 6$, frăgil, separa anteriormente a massa faríngea piriforme $e$ volumosa. Os septos $6 / 7$ $-9 / 10$ são musculosos e espessos, os seguintes delgados. Os 5/6-7/8 partem dos intersegmentos correspondentes, os $8 / 9 \mathrm{e}$ $9 / 10$ tèm a origem dorsal, mas não a ventral, deslocada para 
trás; dorsalmente o septo $8 / 9$ parte de $1 / 2$ IX e $\circ 9 / 10$ do in tersegmento $11 / 12$. A moela tem a forma de um cilindro muitōo musculoso em VI. Três pares de glândulas calcíferas, mais altas do que o esôfago, piriformes e com largo apéndice en tal, partem dorsalmente do esôfago em VII-IX. A estrutura das glândulas é do tipo tubular-composto, com uma cavidade axial ligeiramente excêntrica. A transiçăo esôfago-intestino situa-se em 17/18. O tiflosole inicia-se em XXVI ou XXVII, a parece como uma lámina dorsal, dobrada em $S$ e tão alta quan= to o diâmetro intestinal. o vaso dorsal tem forma de rosá rio, com nódulos segmentares de XVII para diante. Três pares de corações laterais delgados situam-se em VII-IX e 2 pares de coraçōes intestinais volumosos em X-XI. Vaso subneural presente. Em cada segmento há um par de holonefrídios vesicu lados, com nefridióporos em $c d$.

Dois pares de testículos e de funis seminais, envolvidos por sacos testiculares, encontram-se em X e XI. Em cada segmento os sacos fundem-se totalmente na região ventral, elevam-se de cada lado do esôfago e unem-se dorsalmente por delgada ponte. Dois pares de vesículas seminais dobradas situam-se em XI e XII mas, por distenção dos septos, atingem - segmento XVI. Três pares de espermatecas abrem-se em 6/7 $8 / 9$, na linha $c d$. Em cada espermateca (Fig. 2), a ampola ovóide e achatada é 1,5 a 2 vezes mais longa do que o espesso duto. A parede interna da ampola e do duto apresenta numerosas pregas transversais; câmaras seminais faltam. 0 tamanho das espermatecas aumenta do primeiro ao terceiro par.

Considerações

Rhinodrilus jucundus, próximo de $R$. Zakei, distingue-se pela forma das espermatecas, posse de almofadas glandulares em XIII e XVI, relaçōes entre as cerdas e ornamentação das cerdas genitais. 0 nome da nova espécie origina-se da língua latina, jucundus = jovial.

\section{Rhinodrilus motucu Righi, 1971}

Rhinodrilus motucu, Righi, 1971a:21, figs. 29-34; 1972a:257 Rhinodrilus motucu unais Righi, 1971b:7, figs. 5-8. Rhinodrilus garbei cuiabanus Righi, 1984a:101, figs. 35-40.

Material

Brasil, Estado Sergipe: Umbauba, Fazenda Nova Esperança, 7 clitelados, 1 maduro aclitelado e 3 jovens ( $Z U-812$ ) E.R. dos Santos col. 25.VII.1976. Estado Bahia: Una, l clité lado, holótipo de $R$. motucu unais (ZU-140), E. Amante col. 26.III.1969; Itagibá (não Itajubā), l clitelado, parátipo de R. motucu unais (ZU-141), J.Jim col.5.I.1970; Itagibá, Fazen da Pedra Branca, 2 clitelados (ZU-822a-b), J.Jim col. 24. I $^{-}$ 1975; Jequié, 7 clitelados, 15 maduros aclitelados e 10 jovens $(Z U-290)$, J.Jim col. 19.I.1972. Estado Goiás: Poranga tu, 2 clitelados e 3 imaturos, sintipos de $R$. motucu (ZU-10; ZU-85), G.Righi col. 7.I.1967; margem do Rio Tocantins (ca. 
$\left.14^{\circ} \mathrm{S}-48^{\circ} \mathrm{W}\right)$, NE de Uruaçu, 1 clitelado (ZU-818), M. Grempel col. 1.XI.1971. Estado Mato Grosso: Cuiabá, próximo do Rio Coxipó, 4 clitelados, 1 maduro aclitelado e 3 jovens, sintipos de $R$. garbei cuiabanus (ZU-464), G.Righi \& E.Kanner col. 12.I.1978; Poconé, Fazenda Traíra, 2 clitelados e 5 maduros aclitelados (ZU-481), G.Righi \& E.Kanner col. 13.I.1978.

\section{Considerações}

Pelas descrições originais, $R$. curtus e $R$. motucu sāo as 2 únicas espécies do gênero com poros das espermatecas si tuados na linha das cerdas ventrais. Reexaminando o material típico de $R$. motucu, verifiquei que as espermatecas abrem-se na linha dos nefridióporos, portanto em série com as cerdas $c$ ou cd e não $\operatorname{com} a, b$. O reconhecimento deste caráter invalida a distinção de $R$. motucu unais. As diferenças nas relaçōes entre as cerdas são muito pequenas para justificarem a separação subespecífica.

Confrontando o material tipo de $R$. garbei cuiabanus com a descrição original, vejo que a fig. 40 de Righi(1984a) representa os segmentos $X V-X X X V$ e näo como constou, provavel mente porque o segmento $I$, rudimentar e invaginado, não foí considerado na contagem segmentar desta figura. Assim, a for ma e a posição do campo genital masculino de $R$. garbei cuia banus é similar a de $R$. motucu (Righi, 197la, fig. 30), daí a sinonimia proposta.

As diferenças entre $R$. motucu e $R$. garbei restringemse à posição das traves pubertais ( $R$. motucu em XXII - $1 / \mathrm{n}$ XXVII; R. garbei XXI-XXVI) e à posição dos poros masculinos ( $R$. motucu em 23/24;R. garbei em 21/22). Contudo, Michael sen (1926) não está seguro quanto à posiçāo dos poros em gar bei e não estä excluida a possibilidade do segmento $I$, fre qulentemente rudimentar em Rhinodrilus, nāo ter sido contado quando do posicionamento das traves pubertais. Considero as 2 espécies como válidas até o reestudo do material típico ou topotipico de $R$. garbei.

\section{Rhinodrilus marcusae, sp. n.} (Figs. 3-8)

Material

Brasil, Estado Bahia: Porto da Mandioca (pouco acima de Bananeiras, $12^{\circ} 35^{\prime} \mathrm{S}-39^{\circ} 10^{\prime} \mathrm{W}$, margem do Rio Paraguaçu), 6 clitelados (ZU-821A-C), E. Gouvêa col. 26.IV.1980.

\section{Descrição}

Dos 6 exemplares, 5 estão fortemente enovelados e 1 distendido. Comprimento 125-130 mm. No animal distendido o diâmetro em VI e 5,2 mm, decresce até 4,4 $\mathrm{mm}$ em XI e aumenta até $8,4 \mathrm{~mm}$ no clitelo, na região média é $5,4 \mathrm{~mm} e$ na posteri or 4,0 mm. Pigmento falta. Numero de segmentos $389-414$. prostómio tem forma de calota larga, invaginado juntamente com o segmento I. Os segmentos I-III têm numerosos sulcos longitudinais, os demais segmentos preclitelares são eleva - 
dos no equador. Os segmentos posclitelares são uni- ou bianu lares e 2-3 vezes mais curtos do que os anteriores. Clitelo em XIII, XIV-XXVIII $(=15-16)$, anelar, mais espesso dorsalmen te e com sulcos intersegmentares nitidos. Um par de traves pubertais estende-se de XXI, 1/2 XXI - 1/2 XXVII, lateralmen te às cerdas $b$. As traves são largas, espessas e percorridas por um sulco longitudinal circunvoluto (Fig. 5). 0 bordo do clitelo, lateralmente às traves pubertais, é espessado, em alguns exemplares alarga-se lateralmente como un par de ex pansões aliformes, talvez devido a compressōes durante a fixação. Na linha média ventral de XXI-XXVII há uma faixa glan dular rugosa e elevada, com a forma de I e cruzada em cadā segmento por espessamentos equatoriais. As cerdas $a$ e $b$ de XIII-XXVII são envolvidas por uma área diferenciada, circu lar e bastante elevadas em XXI-XXVI.

As cerdas dispöem-se em 8 séries regulares ao longo do corpo, as ventrais são reconhecíveis a partir de $\mathrm{X}$ e as late rais só após o clitelo. Os espaços $a a$ e $a b$ são uniformes até XII, aumentam sucessivamente de XIII-XXI e continuam mais ou menos uniformes para trás. As relaçōes entre as cerdas são na regiäo média (LXXIV-CIII) $a a: a b: b c: c d: d d=10,00: 1,00$ : $13,00: 0,83: 27,00$ e na região posterior (CCCL-CCCLXX) $a a: a b$ : bc: $c d: d d=9,00: 1,00: 10,00: 1,00: 30,00$. As cerdas normais são sigmóides, alongadas, com nódulo distal e a região subapical apresenta 4 séries de 2 marcas semilunares, mais nitidas no lado côncavo. Na região média do corpo o comprimento das cen das ventrais varia de 367-445 $\mu \mathrm{m}$, em média $413 \mu \mathrm{m}$, e das la= terais de 320-383 $\mu \mathrm{m}$, em média $344 \mu \mathrm{m}$. As cerdas ventrais do clitelo transformam-se em cerdas genitais. Seu comprimento varia de 907-1.192 $\mu \mathrm{m}$ e o diâmetro proximal de 36-44 $\mu \mathrm{m}$. Elas sāo retas, com ligeira curvatura na extremidade proximal e os $1 / 2-3 / 5$ apicais são ornamentados por 4 séries de escava ções semilunares, contando-se 6 escavaçōes por série.

Os septos anteriores até $21 / 22$ têm a forma de cones in terpenetrados, os demais são planos. Os septos $6 / 7-9 / 10$ são muito mais espessos e musculosos do que os seguintes. Os $8 / 9$ e 9/10 têm a origem dorsal, mas não a ventral, deslocada para trás; 0 8/9 parte dorsalmente do intersegmento $9 / 10$ e 0 9/10 do segmento XII parietal. A moela situa-se na cavidade de VI mas, devido a forma dos septos, corresponde externamen te aos segmentos XIV-XVIII. Ela tem a forma de um cilindrō espesso e muito musculoso, exceto numa faixa média ventral mais fläcida (Fig. 6). O esôfago anterior abre-se ventralmen te na moela e o esôfago posterior parte da região póstero = dorsal da moela. Três pares de glândulas calcîferas abrem-se lateralmente no esôfago em VII-IX. As glāndulas são reniformes, achatadas antero-posteriormente e com abertura submedia na no inicio da parte mais larga ventral (Fig. 7) Cada glándula tem uma cavidade central ampla que a acompanha por $2 / 3$ de sua extensão, o restante é ocupado pelos tubos calcíferos. A cavidade central é dividida ao meio por uma lámina que se ramifica na periferia (Fig. 8). Os tubos calcíferos são oblíquos e ramificam-se dicotomicamente $1-2$ vezes. Cecos intestinais faltam. O tiflosole inicia-se num volumoso seio sangtíneo em XXVII.Ele aparece como uma lâmina dorsal em for 
ma de $\mathrm{S}$, com altura correspondente a 1/3-1/2 do diâmetro intestinal. O vaso dorsal é nodular de XXI para diante. De XX$X$ o vaso dorsal dobra-se em $S$ e na extremidade anterior da moela divide-se em 3 ramos. O ramo mediano segue dorsalmente até a massa faríngea e cada ramo lateral acompanha o enovela do dos nefridios anteriores até a parede do corpo em $V$ Trés pares de coraçōes laterais delgados encontram-se em VII-IX e 2 pares de volumosos coraçōes intestinais em X e XI. Vaso subneural presente. Em cada segmento hà um par de holonefrí dios vesiculados, com nefridióporos em $c d$. Os nefrídios dé VI e anteriores enovelam-se de cada lado do esôfago anterior. Dois pares de testiculos e de funis seminais prateados situam-se ventralmente em $X$ e XI, envolvidos por sacos testi culares. Os sacos de cada segmento fundem-se ventralmente $\bar{e}$ elevam-se de cada lado do esôfago como una faixa fina que pode fundir-se dorsalmente. Dois pares de vesiculas seminais volumosas encontram-se em XI e XII mas, devido à forma dos septos, ocupam o espaço correspondente aos segmentos XVIII XXI externos. Em vista lateral as vesículas săo dobradas em $\mathrm{V}$, com o ramo dorsal mais largo, recobrindo o esôfago posterior. Três pares de espermatecas abrem-se na linha dos nefri dióporos em 6/7-8/9. Em cada espermateca (Figs. 3-4) nāo hà distinção externa entre duto e ampola, só reconhecíveis em preparaçōes glicerínicas. A ampola é eliptica, achatada dorso-ventralmente e $1,5-2$ vezes mais longa do que o duto. 0 du to é espesso, com numerosas câmaras seminais intraparietais e está merguíhado na espessa parede do corpo. As espermate cas tornam-se maiores do primeiro ao terceiro par.

\section{Considerações}

A nova espécie pertence ao grupo formado por RhinodriZus garbei e $R$. motucu, distinguindo-se pelas relaçöes entre as cerdas e pela forma do campo genital masculino. 0 nome da nova espécie è uma homenagem à Dra. Eveline du Bois-Reymond Marcus.

\section{Rhinodrilus xeabaibus Righi, 1969}

Rhinodrilus xeabaibus Righi, 1969:155, fig. 4.

Material

Brasil, Estado Rio de Janeiro: Itatiaia, 1 clitelado (ZU-816), E. H8fling col. 15.X.1982; Mauä, l clitelado, frag mentado (ZU-817), J Jim col. XII.1966.

\section{Considerações}

0 animal de Itatiaia provavelmente foi anestesiado. 0 clitelo, não túrgido, estende-se de $1 / 2$ XV - 1/2 XXIX (= 14) e expande-se lateralmente na região das traves pubertais. A face ventral de $1 / 2$ XXI - $1 / 2$ XXVIII apresenta alguns sulcos longitudinais. 0 par de largas traves pubertais situa-se lateralmente às cerdas $b$ de XXIII - 1/2 XXVIII, separando-se 
do clitelo por um sulco longitudinal profundo. Sulcos profun dos e circunvolutos percorrem toda a superfície ventral das traves. Papilas genitais circundam as cerdas $a$ e $b$ de VIII XXVIII, devido à proximidade de $a$ e $b$, as papilas se tocam em VIII-XIII, são independentes para träs. 0 animal de Mauá está muito contraído. 0 clitelo ocupa os segmentos XVI-XXIX $(=14)$ e está muito túrgido em XVII-XXVIII, com sulcos intersegmentares nítidos. Devido à contração, a face ventral de XX-XXVIII está muito pregueada longitudinal e transversalmen te e as papilas genitais não são reconhecíveis. 0 par de trā ves pubertais estende-se de $1 / 2$ XXIII - XXVIII.

\section{Urobenus Benham, 1887}

Urobenus Benham, 1887:82; 1890:255; Beddard, 1895:661.

Anteus (part.); Rosa, 1896:90.

Rhinodrilus (part.); Michaelsen, 1900b:430; 1918:165; Ste phenson, 1930:894; Cordero, 1945:8; Righi, 1971a:10; Jamieson, $1971: 738$.

Rhinodrilus (Rhinodrilus) (part.); Cognetti, 1906:174.

\section{Diagnose}

Cerdas dispostas em 8 séries longitudinais regulares

Traves pubertais, quando presentes, intraclitelares. Moela em VI. Três pares de glânduals calcíferas em VII-IX, as de VII e VIII de estrutura tubular-dicotómica (paniculada) no todo ou em sua maior parte, as de IX saculares, com parede fina e ampla cavidade dotada de pregas irregulares e espar sas. Corações em VII-XI, vaso subneural presente. Aparelho genital holândrico e matagínico. Vesículas seminais curtas Um par de poros masculinos intraclitelares. Espermatecas pre sentes, pretesticulares.

Tipo do gênero

Urobenus brasiliensis Benham, 1887.

\section{Consideraçōes}

Devido à fragilidade dos septos anteriores e à sua for ma infundibular, associada ao alongamento da parte anterior do trato digestivo, Benham (1887) interpretou erroneamente a posição segmentar dos órgãos de Urobenus brasiliensis e elegeu o novo gênero. Rosa (1896) corrigiu a descriçäo do genótipo e colocou Urobenus na sinonímia de Anteus, Michaelsen (1900b) transferiu-o para a sinonímia de Rhinodrilus onde permaneceu até hoje. As espécies agora reunidas sob Urobenus distinguem-se de Rhinodrizus principalmente pela estrutura diferencial das glândulas calcíferas de IX. Em algumas espécies de Urobenus as glândulas calcíferas de VII e VIII não partem diretamente do esôfago, mas de evaginações dorsais. 
Lista das espécies com sinonímia e ocorrências.

1. Urobenus brevis (Omodeo, 1955).

Rhinodrilus papillifer f brevis Omodeo, 1955:199, fig. la-c.

Ocorrência - Venezuela: Rancho Grande (Omodeo, 1955).

2. Urọbenus igpigpuera (Righi, 1982)

Rhinodrilus igpigpuera Righi, 1982:111, figs. 8-14.

Ocorrência - Brasil, Estado Pará: Parque Nacional da Amazônia (Righi, 1982).

3. Urobenus petrerei, sp. n.

Ocorrência - Brasil, Estado Maranhão: São Luis.

4. Urobenus gitus (Righi, 1971).

Rhinodrilus gitus Righi, 197la:19, figs. 26-28.

Ocorrência - Brasil, Estado Pará: Belēm.

5. Urobenus buritis (Righi et all. 1976)

Rhinodrilus brasiliensis buritis Righi et all. 1976:352,figs. 28-32.

Ocorrência - Brasil, Estado Amazonas: Manaus (Righi et all. 1976).

6. Urobenus brasiliensis brasiliensis Benham, 1887

Urobenus brasiliensis Benham, 1887:82, est. 8, figs. 11-21, est. 9, fig. 43; 1890:255, fig. 26; Beddard, 1895:661. Urobenus papillifer; Beddard, 1895:662.

Anteus papillifer Michaelsen, 1892:214, est. 13, fig. 4; Rosa, 1896:96, 128 .

Anteus brasiliensis; Rosa, 1896:108, 128.

Rhinodrilus brasiliensis; Michaelsen, 1900b:432; Righi,1971a: 78.

Rhinodrilus papillifer; (part.) Michaelsen, 1900b:432;(part) 1918:181, est. 2, fig. 21; Luederwaldt, 1927:550.

Rhinodrilus (R.) brasiitensis; Cognetti, 1906:175.

Rhinodrilus (R.)papillifer (part.); Cognetti, 1906:175.

Rhinodrilus p. papillifer; Righi, 1971a:78; 1971b:6.

Rhinodrilus b. brasiliensis; Righi, 1974:551, figs. 1-4; 1980a:242.

Ocorrências - Brasil, Estado Minas Gerais: Gorduras (arredores de Belo Horizonte) (Michaelsen, 1918), Conceição do 
Mato Dentro (Righi, 1971b); Estado Rio de Janeiro: Pdza Açu (provavelmente Pedra Açu próximo de Teresópolis) (Benham, 1887), Petropolis, Nova Friburgo (Michaelsen, 1918). Planalto do Itatiaia (Luederwaldt, 1927; Righi, 1974); Est. São Paulo: São Bernardo do Campo (Righi, 1980), Paranapiacaba (= Alto da Serra), Jundiaí (Luederwaldt, 1927), Serra da Cantareira (próximo de Pedra Branca), Botucatu (Righi,1974); Est. Santa Catarina: margens do Rio Itapocu (Michaelsen, 1918), Rio dos Cedros (Alto Palmira) (Righi, 1974); Estadó Rio Grande do Sul: Porto Alegre (Michaelsen, 1892), Itaqui (Luederwaldt, 1927). Paraguai, Departamento Central: San Ber nardino (Rosa, 1896 )

\section{Urobenus brasiliensis teres (Ude, 1894).}

Anteus teres Ude, 1894:59, figs. 6-7.

Urobenus teres; Beddard, 1895:662.

Rhinodrilus papillifer (part.); Michaelsen, 1900b:432. Rhinodrilus (R.) papillifer (part.); Cognetti, 1906:175. Rhinodrilus papillifer var. teres; Michaelsen, 1918:182. Rhinodrilus papillifer teres; Righi, 1971a:78; 1972:156. Rhinodrilus brasiliensis teres; Righi, 1974:555.

Ocorrências - Brasil, Estado Rio Grande do Sul: Taquara (Ude, 1894); Estado Mato Grosso: São José da Serra (Righi,

A descrição de $R$. papillifer var.teres feita por Cernosvi tov (1935) com material do Brasil: Mendez, não permite saber se se trata de $U$. brasiliensis brasiliensis, $U . B$. teres, $U$. buritis ou $U$. brevis.

Chave para as espécies e subespëcies de Urobenus.

1 - Espermatecas, 2 pares em 6/7-7/8.

-- Espermatecas, 3 pares em 6/7-8/9.

2 - Espermatecas com câmaras seminais.

--- Espermatecas sem câmaras seminais.

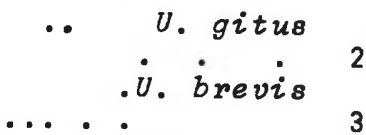

3 - Glândulas calcíferas de VII e VIII subdivididas: parte proximal arredondada e de estrutura tubular-compos ta; parte distal, principal, piriforme e de estrutura tubular-dicotômica.

-- Glândulas calcíferas de VïI e vïí nã̃o subdivididas e de estrutura tubular-dicotômica.

4 - Septos anteriores espessados. Cecos intestinais ausen tes. $\quad . \quad: \quad . . . \quad$. . igpigpuera

--- Septos anteriores frägeis. Cecós intestinais presen -

5 - Poros masculinos em $2 \ddot{0} \ddot{2} i$.

. $\dot{U}$ buritis

-- Poros masculinos em $19 / 20$...

6 - Traves pubertais em XIX-XXII, I/2 XẌİ̈.

-.- Traves pubertio..... … brasiliensis

\footnotetext{
.U. brasiliensis teres
.
} 
Urobenus petrerei, sp. n. (Figs. 9-13)

Material

Brasil, Estado Maranhão: São Luis, 13 clitelados e 33 jovens $(Z U-8 I 1)$, M. Petrere col. V.1984.

Descrição

Comprimento 67-73 mm. Diâmetro na região média 4,1-4,2 $\mathrm{mm}$, pouco variável ao longo do corpo. Número de segmentos 126-128, todos bianelares. Segmentos I-II rudimentares, de parede flácida, sem pigmento, o I sempre invaginado, junta mente com o prostômio tentaculiforme. Cor no dorso marrom avermelhada como a do n? 705 de Séguy (1936), ventre esbran quiçado, o limite entre as cores e brusco na linha dos nefri dióporos. Clitelo em sela nos segmentos XIV- $1 / 2 \mathrm{XXV} \quad(=1 \bar{I}$ 1/2), ligeiramente espessado e de cor marrom clara como a do n? 695. Outras marcas pubertais faltam. Cerdas dispostas em 8 séries longitudinais regulares. Na região média do corpo, segmentos $X L-L$, as relações entre as cerdas são $a a: a b: b c: c d$ : $d d=7,6: 1,0: 5,5: 0,8: 26,6$ e na região posterior, segmentos $c-c X, a a: a b: b c: c d: d d=5,5: 1,0: 5,0: 0,8: 16,6$. As cerdas ventrais do clitelo não estao transformadas em genitais. Na região média as cerdas são sigmóides, com nódulo distal e o 1/7 apical ornamentado por marcas semilunares, côncavas para - ápice e dispostas sem regularidade entre si. 0 comprimento das cerdas ventrais varia de 490 a $570 \mu \mathrm{m}$, em média $528 \mu \mathrm{m}$, e as cerdas laterais variam de 384-490 $\mu \mathrm{m}$, em média $450 \mu \mathrm{m}$.

Os septos 6/7-11/12 são bastante espessos e musculo sos, dispostos como cones interpenetrados, os seguintes são frágeis e planos. Uma moela globóide e muito musculosa situa -se em VI e 3 pares de glândulas calcíferas em VII-IX. Cada glândula de VII e VIII compöem-se de uma porção proximal (Fig. 9, GCP), dorsal ao esófago e de contorno arredondado, que se comunica lateralmente, através de pequeno estrangulamento, percorrido por um duto ciliado, com uma porção distal (GCD) cerca de 3 vezes mais volumosa, em forma de retorta química com pequena dilatação terminal. A porção proximal apresenta uma ampla cavidade central e várias pregas parie tais largas e irregularmente interligadas, deixando entre si espaços periféricos largos e pouco ramificados (Fig. $10 \mathrm{GCP}$ ), corresponde à estrutura tubular-composta (Kompositenschlauch tasche) de Michaelsen (1918) A porção distal é tipicamenté tubular-dicotômica (Rispenschlauchtasche), a cavidade ectal é curta, logo subdividida num sistema de túbulos longitudi nais pouco ramificados (Fig. 10, GCD; 11) Na porção proxi mal a capa muscular parietal é mais espessa do que na distal. As porções proximais das glândulas simetricas fundem-se en tre si pouco antes de abrirem en comum numa expansão dorsal do esôfago nesses segmentos. O par de glândulas de IX tem forma e estrutura diferente. Cada glândula abre-se dorso-lateralmente na região posterior do esôfago através de um duto longo, dotado de cílios altos. Estas glândulas são renifor mes (Fig. 12, GC) com a parte dorsal prolongando-se num apên 
dice curto, estreito e dobrado para trás. Nos cortes as glândulas aparecem como largos sacos vazios, com raras pre gas parietais irregulares, mais altas e numerosas nas proximidades da abertura. 0 intestino inicia-se em XVI. 0 tifloso le aparece bruscamente em XXVI, apresenta-se como uma lâminà ligeiramente ondulada e de altura equivalente a $1 / 2$ do diâme tro intestinal. Um par de cecos intestinais pequenos e arredondados, nem sempre reconhecíveis, situa-se de cada lado da linha média ventral em XXVI. De XVI-XXVI hä 3 pares de vasos dorso-intestinais por segmento. 0 vaso dorsal, retilíneo posteriomente, tem aspecto moniliforme e dobrado segundo un $S$ apertado entre XVII e XIII. Vaso subneural presente. Dois pares de corações intestinais volumosos estão em X e XI e 3 pares de delgados corações laterais em VII-IX. Em cada segmento há um par de holonefrídios com nefridióporos intersegmentares na linha das cerdas $d$. Os nefrídios de VI e ante riores enovelam-se dos lados da regiāo anterior do esôfago.

Dois pares de testículos e de funis seminais pequenos situam-se ventralmente em $X$ e XI, livres. Dois pares de vesí culas seminais arredondadas encontram-se latero dorsalmente em XI e XII, o par posterior pode salientar-se em XIII, XIV ou XV, atravessando como tubo os delgados septos anteriores. Um par de ovários e de funis femininos encontram-se em XIII. Três pares de espermatecas abrem-se na linha dos nefridióporos em 6/7-8/9 e salientam-se nas cavidades de VII-IX. As es permatecas (Fig. 13) são claviformes, sem distinçāo entre. dū to e ampola e tornam-se maiores do primeiro ao terceiro par. O nome da nova espécie foi dado em homenagem ao $\mathrm{Dr}$. Mi guel Petrere, que coletou os animais.

\section{REFERENCIAS}

BEDDARD, F E., 1892. The earthworms of the Vienna Museum Ann.Mag.nat.Hist. (6) 9:113-134, est. 7

BEDDARD, F E., 1895. A monograph of the order of oligochaeta. XII + 769 pp. 5 est. Clarendon Press, Oxford.

BENHAM, W. B., 1887 Studies on earthworms. II.Quart.J.micr. Sc. (N.S.) 27:77-108, est. 8-9.

BENHAM, W. B., 1890. An attempt to classify earthworms Quart.J.micr.Sc. (N.S.) 31:201-315.

CERNOSVITOV, L., 1934. Les Oligochètes de la Guyane Française et d'autres pays de l'Amerique du Sud. BulZ.Mus.Nat. Hist.nat.Paris (2) 6:47-59.

CERNOSVITOV, L., 1935. Oligochaeten aus dem tropischen SUd Amerika. Capita Zool., $6(1): 1-36$, est. 1-6.

CERNOSVITOV, L;, 1938. Deux nouveaux Oligochètes Glossoscole cides du Brésil. Bulz.Assoc.philom. Alsace et Lorraine, 1938:401-407.

COGNETTI DE MARTIIS, L., 1902. Viaggio del Dr. A. Borelli nel Chaco Boliviano e nella Republica Argentina. Terricoli boliviani ed argentini. Boll.Mus.Torino, 17(420):1-11, 1 est.

COGNETTI DE MARTIIS, L., 1906. Gli Oligochaeti della Regione Neotropicale. II. Mem.R.Accad.Sc.Torino (2) 56:147-262, 2 est. 
CORDERO, E. H., 1943. Oligoquetos Sudamericanos de la Fami lia Glossoscolecidae, II. Dos nuevas especies de Rhinodri lus del nordeste del Brasil. Comun.zool. Mus. Hist.nat.Montevideo, $1(6): 1-6$.

CORDERO, E. H., 1944a. Oligoquetos Sudamericanos de la Familia Glossoscolecidae, III. Rhinodrilus francisci, n. sp., de Pernambuco, Brasil. Comun.zool. Mus. Hist.nat.Montevideo, $1(10): 1-4$.

CORDERO, E. H., 1944b. Oligoquetos Sudamericanos de la Familia Glossoscolecidae, IV Sobre algunas especies de Venezuela. Comun.zool. Mus.Hist.nat.Montevideo, 1(14):1-6.

CORDERO, E. H., 1945. Oligoquetos Sudamericanos de la Fami lia Glossoscolecidae, VI. Los generos de la Subfamilia Glossoscolecinae, sus probables relaciones fileticas y su distribucion geográfica actual. Comun.zooi.Mus.Hist.nat. Montevideo, 1(22):1-28, 1 est.

HÖLING, E., 1980. Sistema circulatório de Rhinodrilus ala tus Righi, 1971 (Oligochaeta, Glossoscolecidae) Papéis Avulsos Zool., S. Paulo, 34(3):21-72.

HOFLING, E., 1982 Capilarização superficial de Rhinodrilus alatus Righi, 1971 (Oligochaeta, Glossoscolecidae) $\mathrm{Pa}$ péis Avulsos Zool., S.Paulo, 34(26):297-307.

HORST, R., 1891. Descriptions of earthworms, VI. Notes Ley den Museum, 13:77-84, est. 6 .

JAMIESON, B. G. M., 197i. Family Glossoscolecidae. In Brin khurst, R. 0. \& Jamieson, B. G. M., Aquatic oligochaeta of the world: 723-837. Oliver \& Boyd, Edinburgh.

LUEDERINALDT, H., 1927 A colleção de minhocas (Oligochaeta) do Museu Paulista. Rev.Mus.Paulista, 15:545-556.

MICHAELSEN, W., 1892. Terricolen der Berliner zoologischen Sammlung, II. Arch. Naturg., 58(1):209-261, est. 13.

MICHAELSEN, W., 1895. Zur Kenntnis der Oligochaeten. Abhand. naturw. Ver. Hamburg, 13:1-37, 1 est.

MICHAELSEN, W., 1900a. Die Terricolen-Fauna Columbiens.Arch. Naturg., 66:231-266.

MICHAELSEN, W., 1900b. Oligochaeta. Das Tierreich, 10:XXIX + 575 pp. R. Friedlunder und Sohn, Berlin.

MICHAELSEN, W., 1918. Die Lumbriciden mit besonderer Be rllcksichtigung der bisher als Familie Glossoscolecidae zusammengefassten Unterfamilien. Zool.Jb.Syst., 41:1-398, est. $1-2$.

MICHAELSEN, W., 1926. Zur Kenntnis einheimischer und auslandischer Oligochyten. Zool.Jb.Syst., 51:255-328.

MICHAELSEN, W., 1928. Miscellanea Oligochaetologica. Ark. f8r Zool., $20(2): 1-15$.

MICHAELSEN, W., 1931. Zwei neue aussereuropalische Oligochyten des Senckenberg Museums. Senckenbergiana, 13:78-86.

MICHAELSEN, W., 1934. Opisthopore Oligochaten des K8nigli chen Naturhistorischen Museums von Belgien. BulZ.Mus.R. Hist.nat. Belgique, 10:1-29.

MICHAELSEN, W., 1935. Die opisthoporen Oligochaten Westin diens. Mitt.zool. Yus. Hamburg, 45:51-64.

OMODEO, P., 1955. Oligocheti Terricoli del Venezuela raccolti dal dr. Marcuzzi. Mem.Mus.civ.Stor.nat.Verona, 4:199212 . 
PANTIN, C. F A., 1964. Notes on microscopical technique for zoologists. VIII + 77 pp. University Press, Cambridge.

PERRIER, E., 1872. Recherches pour servir a l'histoire des lombriciens terrestres. Nouv.Arch.Mus.Hist.nat.Paris, 8 (1) : 5-198, est. 1-4.

RIGHI, G., 1967. Descrição de Rhinodrilus priollii, sp.n. Glossoscolecidae da Amazônia, com bibliografia dos 0ligochaeta terrícola da regiāo. Atas Simpōsio Biota Amazôni ca, 5 (Zool.): 475-479.

RIGHI, G., 1969. On some Brazilian Glossoscolecidae. Beit. neotrop. Fauna, 6(2):152-162.

RIGHI, G., 1971a. Sobre a Familia Glossoscolecidae (Oligo chaeta) no Brasil. Arq.Zool., S.Pauzo, 20(1):1-96.

RIGHI, G., 1971b. Sobre alguns 0ligochaeta brasileiros. Papéis Avulsos Zool., S.Paulo, $25(1): 1-13$.

RIGHI, G., 1972a. Bionomic considerations upon the Glossosco lecidae (Oligochaeta). Pedobiologia, 12:254-260.

RIGHI, G., 1972b. Contribuiçāo ao conhecimento dos oligochae ta brasileiros. Papéis Avulsos zool., S.Paulo, $25(18): 149^{=}$ 166.

RIGHI, G., 1974. Notas sobre os Oligochaeta Glossoscolecidae do Brasil. Rev. Brasil. Biol., 34(4):551-563.

RIGHI, G., 1980a. Alguns Oligochaeta, Ocnerodrilidae e Glossoscolecidae do Brasil. Papéis Avulsos Zool., S.Paulo, 33 (13) : 239-246.

RIGHI, G., $1980 \mathrm{~b}$. Alguns Megadrile (Oligochaeta, Annelida) brasileiros. Bolm.Zool., Univ.S.Paulo, 5:1-18.

RIGHI, G., 1982. Oligochaeta Glossoscolecidae do Parque Na cional da Amazônia, Tapajós. Rev.Brasiz.Biol., 42(1):107116.

RIGHI, G., 1984a. On a collection of Neotropical Megadrili $\underline{0}$ ligochaeta. II. Glossoscolecidae, Lumbricidae. Stud. Neotrop. Fauna, $19(2): 99-120$.

RIGHI, G., 1984b. Nova contribuiçāo ao conhecimento dos 0ligochaeta da Venezuela. Papéis Avulsos Zool., S.Paulo, 35 (2) : 243-256.

RIGHI, G., 1984c. Oligochaeta Megadrili da região centro-oes te de Mato Grosso, Brasil. Bolm.Zool., Univ.s.Paulo, 8 : 189-213.

RIGHI, G., I. AYRES \& E. C. R. BITTENCOURT, 1976. Glossoscolecidae (0ligochaeta) do Instituto Nacional de Pesquisas da Amazônia. Acta Amazōnica, 6(3):335-367.

ROSA, D., 1895. Viaggio del dottor Alfredo Borelli nella Republica Argentina e nel Paraguay. XV Oligocheti Terrico1i. Boll.Mus. Torino, $10(20): 1-3$.

ROSA, D., 1896. Contributo allo studio dei terricoli neotropicali. Mem.R.Accad.Sc.Torino (2) 45:89-152, 1 est.

SCHNEIDER, G., 1892. Ueber eine neue Regenwurmart aus Trinidad. SB.Ges.Dorpart. 11:42-44 (não visto, citado de Bed dard, 1895 ).

SCHROEDER-ARAUJO, L.T, 1978. Topografia vascular de Rhino drilus duseni Michaelsen, 1918 (Oligochaeta, Glossoscolecidae) Bolm.Zool., Univ.s.Paulo, 3:163-199.

SEGUY, E., 1936. Code universel des couleurs. 68 pp. 55 est. Paul Lechevalier ed., Paris. 
STEPHENSON, J, 1930. The Oligochaeta. XVI + 978 PP. Clarendon Press, Oxford.

STEPHENSON, J , 1931. Oligochaeta from Burma, Kenya and other parts of the world. Proc.zool.Soc.London, 1931:33 92.

UDE, H., 1894. Beitrlage zur Kenntnis auslalndischer Regenwllrmer. Zeit.wiss.200l., 57:57-75, est. 4 .

VAILLANT, L., 1889. Histoire naturelle des Annelés marins et d'equ douce. III. Lombriciens, Hirudiniens, Bdellomorphes, Térétulariens et Planariens. Paris (não visto, citado de Beddard, 1895).

\section{Estampa 1}

Rhinodrilus jucundus .

Fig. 1 - Face ventral dos segmentos XII-XXVIII.

Fig. 2 - Espermateca de 8/9, preparação glicerínica.

Rhinodrilus marcusae.

Fig. 3 - Espermateca de 6/7, preparação glicerínica.

Fig. 4 - Idem de $8 / 9$.

Fig. 5 - Face ventral dos segmentos XVII-XXXII.

Fig. 6 - Moela em vista lateral. 


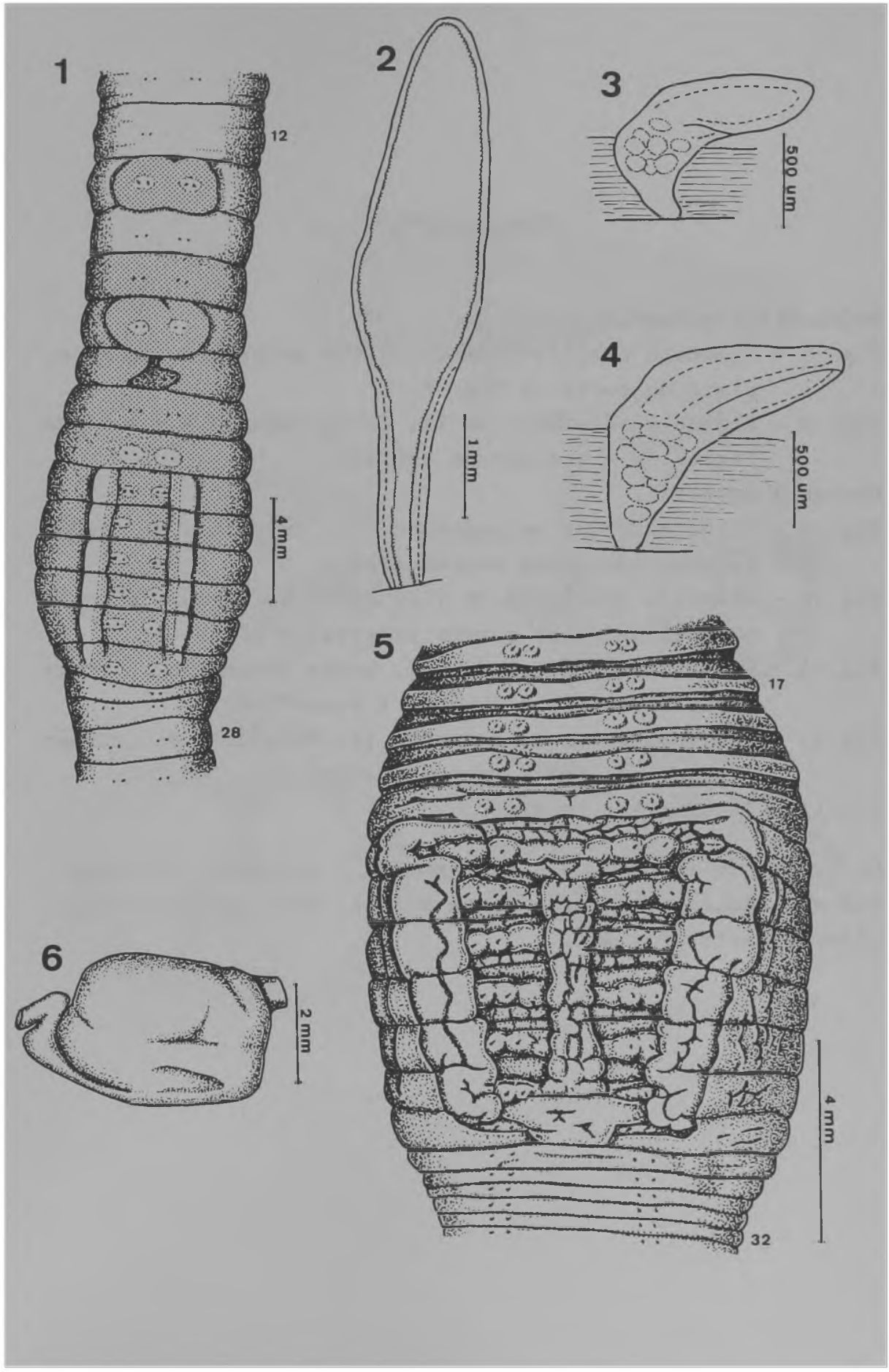




\section{Estampa 2}

Khinodrilus marcusae.

Fig. 7 - Glândula calcífera de IX, vista anterior. a - a, plano do corte da Fig. 8.

Fig. 8 - Glândula calcífera de IX, corte segundo a - a da Fig. $7 \mathrm{X}$ - posição do esôfago.

Urobenus petrerei.

Fig. 9 - Vista anterior do segmento VII. Nefrídios e cordão nervoso não foram representados.

Fig.10 - Glândula calcífera de VII, corte na região de comunicação entre as partes proximal e distal.

Fig.ll - Glândula calcífera de VII, parte distal em corte transversal, algo oblíquo e submediano.

Fig.12 - Vista anterior do segmento IX. Nefrídios e cordão nervoso não foram representados.

Fig.13 - Espermateca de $8 / 9$.

CL - coração lateral. E - esôfago. GC - glândula calcífera.

GCD - glândula calcífera, parte distal. GCP - glândula cal cifera, parte proximal. 

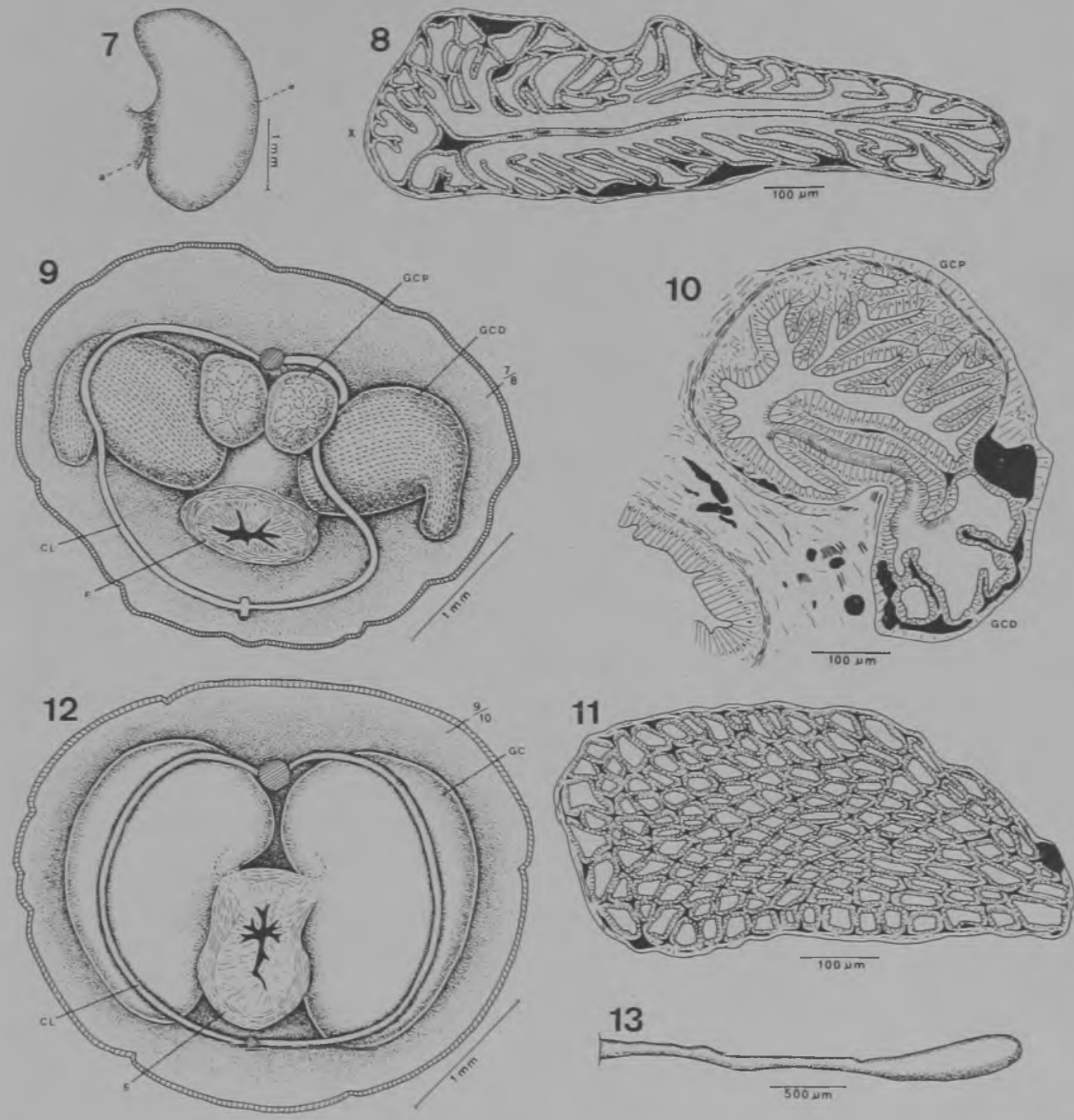

13

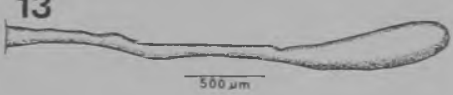


